\title{
Lateral Nasal Capillary Haemangioma a Rare Occurrence-Case Report and Literature Review
}

\author{
Shuaib Kayode Aremu1 ${ }^{*}$, Olajide Toye Gabriel1 ${ }^{1}$, Adewoye Kayode Rasaq ${ }^{2}$, Abdulwasiu Salman ${ }^{3}$ \\ ${ }^{1}$ ENT Department, Federal Teaching Hospital Ido-Ekiti/Afe Babalola University, Ado-Ekiti, Nigeria \\ ${ }^{2}$ Community Medicine Department, Federal Teaching Hospital Ido-Ekiti/Afe-Babalola University, Ado-Ekiti, Nigeria \\ ${ }^{3}$ ENT Department, Federal Teaching Hospital, Ido-Ekiti, Nigeria \\ Email: *shuaib.aremu@gmail.com
}

How to cite this paper: Aremu, S.K., Gabriel, O.T., Rasaq, A.K. and Salman, A. (2018) Lateral Nasal Capillary Haemangioma a Rare Occurrence-Case Report and Literature Review. Case Reports in Clinical Medicine, 7, 567-575.

https://doi.org/10.4236/crcm.2018.711051

Received: October 19, 2018

Accepted: November 13, 2018

Published: November 16, 2018

Copyright (c) 2018 by authors and Scientific Research Publishing Inc. This work is licensed under the Creative Commons Attribution International License (CC BY 4.0).

http://creativecommons.org/licenses/by/4.0/

\section{Open Access}

\begin{abstract}
Nasal lobular capillary haemangioma $(\mathrm{CH})$, also known as pyogenic granuloma, is a benign lesion of unknown etiology commonly associated with pregnancy, oral contraceptive pills, and trauma. Marked by epistaxis and nasal obstruction, the condition involves benign capillary proliferation with a microscopically distinctive lobular architecture, affecting the skin and mucous membranes of the oral cavity and nasal region. This aim of this article is to report a case of lateral nasal capillary haemangioma in a 39-year-old female, who presented in our facilities having experienced spontaneous epistaxis on the left side of the nose for the previous three months. Endoscopy revealed a protruding left intranasal mass arising from the lateral nasal wall in the region of the middle turbinate and osteo-meatal complex. The patient underwent endoscopic excision of the mass and did excellently well thereafter with no recurrence.
\end{abstract}

\section{Keywords}

Haemangioma, Nasal Mass, Endoscopic Excision, Vascular Lesions

\section{Introduction}

Nasal lobular capillary haemangioma $(\mathrm{CH})$, formerly known as pyogenic granuloma is an acquired benign vascular proliferation of unknown origin, with a characteristic lobular architecture on microscopy [1]. The term "lobular capillary haemangioma" was proposed to replace the original name since it more accurately addresses the characteristic histological features of the lesion, which is distinguished by anastomosing capillary networks contained in distinct lobules 
within oedematous and fibroblastic stroma [2]. The stroma may also be fibromyxoid or -oedematous with acute to chronic inflammation. The condition usually affects the skin of the head and neck and mucous membranes of the oral cavity. The condition rarely occurs in juvenile nasal cavities [3]. $\mathrm{CH}$ is a benign lesion which is commonly associated with pregnancy, oral contraceptives, and trauma; and symptomatically marked by epistaxis and nasal obstruction [4]. $\mathrm{CH}$ is still not well-recognized among otolaryngologists and its occurrence is probably underestimated. A large amount of the information found in scientific literature relates to individual case reports [5] [6] [7].

The cervical-facial district is the area most commonly affected by haemangiomas, where the lesions are localized mainly in the gingiva, oral mucosa, tongue, and lips [8] [9] [10]. Generally, haemangiomas arise from a highly vascularized area of the anterior nasal septum known as the locus Valsalva [8], from the nasal vestibule [3] [9], or the lateral wall of the nasal cavity [11]. Scientific literature also reports the incidence of intraosseous haemangiomas, found in the inferior turbinate [12] of the maxillary sinus, the floor of the nasal cavity, and the roof of the vestibule [13]. Cases associated with a gradually developing painless swelling and nasal deformity has also been reported [9]. The volume of sino-nasal haemangiomas is variable, but generally below $10 \mathrm{~mm}$, however, it is possible for a tumor to grow to $80 \mathrm{~mm}$; known as giant haemangiomas [10].

The case is reported in other to highlight the etiology, clinical presentation and treatment of lateral nasal capillary haemangioma and to add to the world literature our own findings. The literature review was done by Electronic searches of the following databases: Web of Knowledge, Web of Science, Google Scholar, PubMed, Cumulative Index to Nursing and Allied Health Literature (CINAHL) and the digital library JSTOR using key words such as capillary haemangioma, nasal mass, endoscopic excision and nasal surgery.

\section{Case Report}

A 39-year-old female with a complain spontaneous epistaxis from the left side for three months this year. The patient reported presyncope in association with the aforementioned symptoms but had not experienced a fainting attack nor loss of consciousness.

The patient had not experienced trauma to the nose, had no family history of a bleeding disorder, and was not on any anti-platelet drugs. However, she was in the final trimester of her first pregnancy and had undergone a myomectomy eleven months prior to the onset of epistaxis. As a result, she had spent 48 hours in a private hospital to arrest the bleeding with anterior nasal packing. She subsequently had a cesarean section and delivered of a dead fetus. Approximately a month following delivery, she noticed mild frontal headaches and left nasal "fullness," observable with a mirror as a swelling in the nostril about the size of her distal little finger. This swelling continued to grow for three months till when she presented to the Accidents \&Emergencies of our hospital. 
The patient had no history of swelling in other parts of the body, nor loss of weight, and no anorexia. She did not exhibit any ontological nor throat symptoms, nor did she have a history of nasal allergy, aspirin intolerance, or asthma. The patient is not hypertensive and has no history of pregnancy-induced hypertension, Diabetes Mellitus, Peptic Ulcer Diseases, or previous nasal surgery. She didn't have a history of known drug allergies, nor cigarette smoking and doesn't drink alcohol. The physical examination concluded the patient was quite conscious, not pale, anicteric nor febrile. Other physical symptoms were as follows: PR 76bpm, BP 110/70 mmHg, RR $18 \mathrm{cpm}$. Nasal examination with 0 and 45-degree telescope revealed a protruding left intranasal mass arising from the lateral nasal wall in the region of the middle turbinate and osteomeatal complex (Figure 1) extending to the anterior nares with a central necrotic surface surrounded with bloodstained mucus narrowing the nasal cavity. Both middle and inferior turbinates were hyperaemic and engorged on the right with partly patent nostril. Facial asymmetry was evident, with a left bulging of the nasal pyramid without an increase in medial intercanthal distance. The oral cavity and oropharynx were essentially normal. X-ray of Para-nasal sinuses (Figure 2 and Figure 3) showed bilateral engorged turbinates with opacification in the left nasal cavity and septal deviation to the right. Due to financial constraints, a Computerized Tomographic Scan was not performed. PT/PTTK/INR and other ancillary investigations done were within normal limits.

After informed consent, the patient underwent endoscopic excision of the mass (Figure 4) with cauterization of the base. The histology report (Figure 5) showed fairly circumscribed lesions composed of densely packed small, thin-walled vascular channels set with an oedematous, abundant fibro-collagenous stroma. Areas of hemorrhage, fibrin clots, and patchy infiltrate of the mononuclear inflammatory cell were also seen. The overlying epithelia showed focal hyperplasia in keeping with capillary haemangioma. The patient had excellent postoperative recovery and has not experienced nasal adhesion or recurrence in the month after surgery.

\section{Discussion}

The first description of Lobular capillary haemangiomas was in 1897 by Poncet

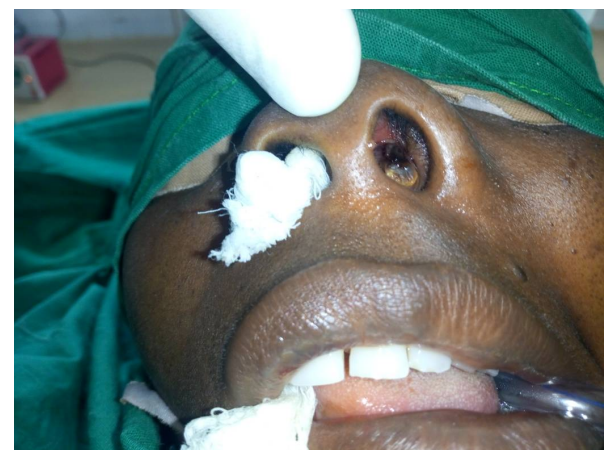

Figure 1. Left Intranasal mass. 


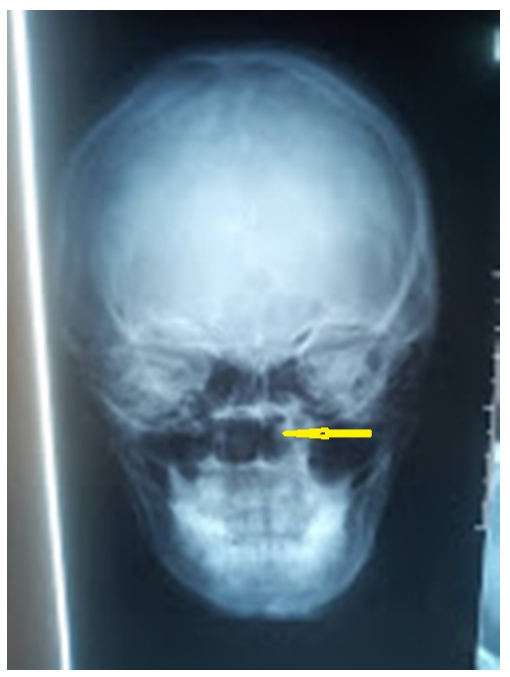

Figure 2. Left intranasal mass.

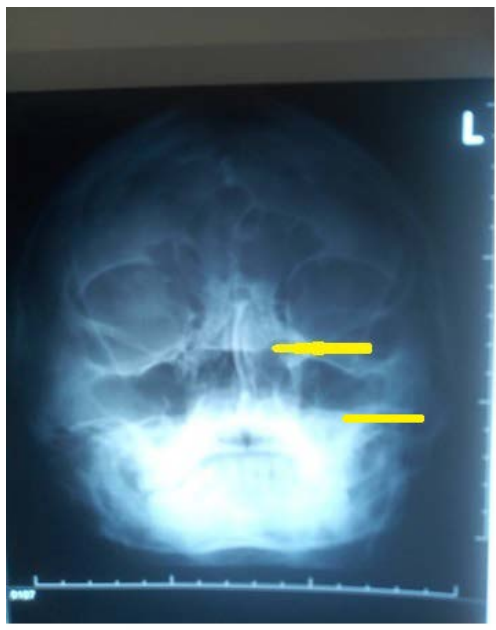

Figure 3. Upper arrow points to Left Intranasal soft tissue shadow with fluid level (lower arrow) in left maxillary sinus.

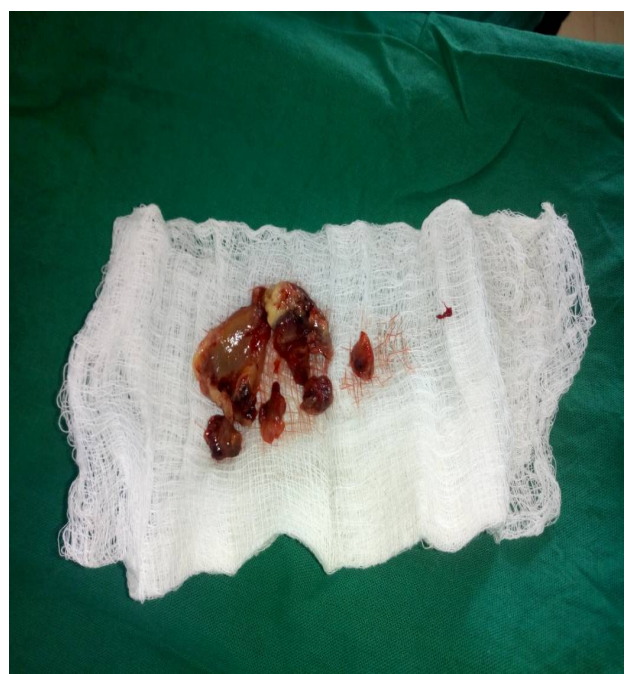

Figure 4. Gross appearance of the tumor. Reddish, fleshy, firm and well-encapsulated mass. 


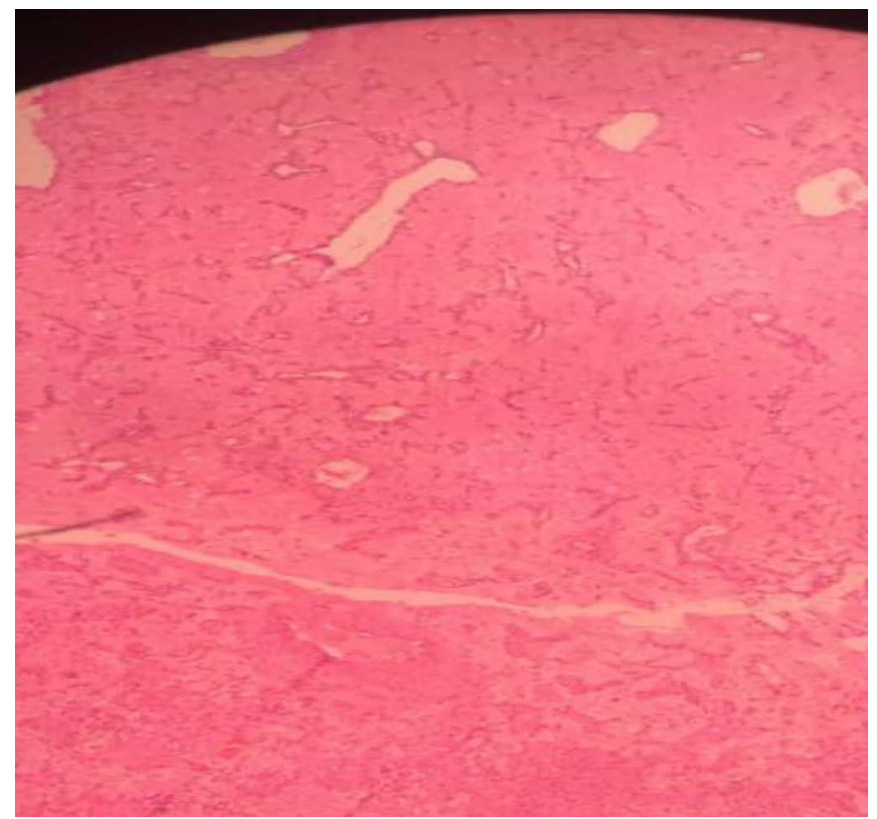

Figure 5. Histology appearance of the mass. Densely packed small, thin-walled vascular channel set with an oedematous, abundant fibro-collagenous stroma (100×, H \& E).

and Dor as botryomycosis hominids, small vascular tumors seen in the fingers of four of their patients, and believed it was secondary ailment to fungal infection [14]. Later, the condition was rediscovered, this time in the nasal cavity, by Frank and Blahd in 1940 [14], and termed as pyogenic granuloma: a name which has also been superseded as the tumors are neither infectious nor granulomatous. Later still, Mills et al. proposed the term lobular capillary haemangioma as a substitute to pyogenic granuloma, due to the characteristic microscopic features of the condition [15].

Pathogenesis of $\mathrm{CH}$ remains unclear, though some evidence supports nasal trauma [16], endocrine disorders [17] [18], viral oncogenes, arteriovenous malformations, and angiogenic growth factors [19]. Increased levels of estrogen and progesterone have been indicated as the hallmark in the pathogenesis of an "a pregnancy tumor;" a specific form of $\mathrm{CH}$ of the mucosa occurring during pregnancy: this pathology is observed in the gingiva and, less often, in the nasal cavity [20]. Furthermore, a higher incidence of "pyogenic granuloma" has been linked to oral contraceptives with greater progestinic activity [20].

The frequent location of $\mathrm{CH}$ at the anterior nasal septum (Kiesselbach's area) in recurrent nose pickers or patients with a history of nasal packing lends credence to the belief that trauma localized in this area may precede the genesis of $\mathrm{CH}[21]$.

Mulliken and Glowacki differentiated haemangiomas into three subtypescapillary haemangioma (originating predominating from the hypervascular septal region, locus Valsalva), cavernous haemangioma (generally found at the side wall of the nasal cavity), and mixed forms-under the broad umbrella of fastgrowing tumors [11]. 
The characteristic microscopic appearance of capillary haemangiomas is the presence of a central lobular region, histologically formed by a capillary network with vascular dilation, inflammation, and granulation [22] [23].

Endothelial cells, pericytes, myeloid cells, fibroblasts, and plasma cells characterize the cytological structure and the central lobular region is easily distinguishable in radiologic studies with contrast due to the rich vascularization of the area [9] [24]. Some of the key aspects of haemangioma can be evaluated in a CT scan, as demonstrated by using this method of radiography; there is a clear contrast in the lobular region due to the intensity of soft tissue [24]. Although the surface portion of a tumor does not contrast well in CT scans, this imaging technique rates better than any other radiological examination for analyzing the original bony architecture of the sino-nasal cavity and for identifying possible bone erosion; as is common at the base of $\mathrm{CH}$ lesions [24]. Imaging studies are frequently used to evaluate the differential diagnosis and surgical possibilities [25]. CT imaging is the modality of choice in investigating LCH. It has the non-specific features of a well-defined soft tissue density mass with a hypoattenuating cap of variable thickness [25]. Contrast-enhanced CT scans of the paranasal sinuses are the usual mode of examination used for imaging lobular nasal capillary haemangiomas and are expected to reveal a soft-tissue enhancing mass (bony destruction at the root of a tumor may or may not be present [25]). A CT scan without contrast enhancement should show a well-defined soft-tissue mass able to undergo intense enhancement on postcontrast study [25]. Generally, bony erosion is not associated with lobular nasal capillary haemangiomas, though a few studies do find this symptom to be a characteristic [26]. Bony erosion of the medial left antral wall was observed in our patient [26].

CT scans are extremely useful for clinical analysis, enabling medical professionals to rule out various further symptoms of $\mathrm{CH}$, including erosion of bony structures [26], invasion of adjacent paranasal sinuses or intracranial extension. The most common differential diagnosis from CT scans is nasal angiofibroma [26]. A T2-weighted MRI of $\mathrm{CH}$ would show a number of flow voids running through vascular tissue [26].

The most common presenting symptoms of $\mathrm{CH}$ of the nasal cavity include recurrent unilateral epistaxis and nasal obstruction; facial pain, alteration of smell, and headache are seldom observed [25]. At endoscopy, the lesion usually appears as a single hypervascularized mass of a red to purple color, not exceeding $1 \mathrm{~cm}$, and with a predilection for the anterior portion of the nasal septum. However, many $\mathrm{CH}$ also frequently present as a mass of considerable size, entirely filling the nasal cavity: for such lesions, the term "giant LCH" has been coined. Diagnosis of $\mathrm{CH}$ in its usual presentation-a small hypervascularized mass localized anteriorly in the nasal cavity-is not problematic. However, differential diagnosis may pose challenging problems in larger lesions, as shown by the fact that in these study three cases of $\mathrm{CH}$ were initially misdiagnosed as either angiofibroma or in one case, a low-grade angiosarcoma [25]. 
The histopathological appearance of $\mathrm{CH}$ is characterized by circumscribed anastomosing capillary networks, arranged in the oedematous and fibroblastic stroma in a lobular architecture. According to Toida et al. [25], in most cases it was possible to distinguish a lobular area and a superficial ulcerative area. Specifically, this haemangioma in this case study demonstrated atypical proliferation of microvascular elements in the lobular area, and the ulcerative area showed superficial neutrophilic infiltrates and irregular dilatation of blood vessels [26] [27].

Surgery is the treatment of choice for this condition [28], while the conservative endoscopic excision is the preferred treatment for $\mathrm{CH}$ of the nasal cavity. Surgical methods used include electrocoagulation, cryotherapy, LASER, excisional surgery, and excisional surgery following angiography with embolization [21]. Surgical excision was what we did for the index patient and she did very well. She was followed up for 6 months without feautes of recurrence. The recurrence rate for haemangioma ranges from $0 \%$ to $42.0 \%$ [21] [29].

Pregnancy-related nasal lobular capillary haemangioma may self-resolve following childbirth, though surgery may be required for postpartum lesions that do not completely resolve. The etiology of the lesion in the patient described above may also be pregnancy-related [30]. The case is reported since this condition has a high potential for misdiagnosis, and to highlight the advantage of nasal endoscopes in diagnosis and treatment.

\section{Conclusion}

Capillary haemangioma is a common condition affecting various regions of the body. Localization in the nasal cavity is not often reported thus believed to be quite rare. The case is reported since this condition has a high potential for misdiagnosis and to highlight the importance of using nasal endoscope in the examination of any epistaxis patient with the regular follow up.

\section{Conflicts of Interest}

The authors declare no conflicts of interest regarding the publication of this paper.

\section{Consent}

Informed consent was obtained from the patient before writing this case report.

\section{Ethical Approval}

This was obtained from the ethical committee of our hospital before writing this case report.

\section{References}

[1] Patrice, S.J., Wiss, K. and Mulliken, J.B. (1991) Pyogenic Granuloma Lobular Capillary Hemangioma: A Clinicopathologic Study of 178 Cases. Pediatric Dermatology, 
8, 267-276.

[2] Mills, S.E., Cooper, P.H. and Fechner, R.E. (1980) Lobular Capillary Hemangioma: The Underlying Lesion of Pyogenic Granuloma. A Study of 73 Cases from the Oral and Nasal Mucous Membranes. The American Journal of Surgical Pathology, 4, 470-479. https://doi.org/10.1097/00000478-198010000-00007

[3] Katori, H. and Tsukuda, M. (2005) Lobular Capillary Hemangioma of the Nasal Cavity in a Child. Auris Nasus Larynx, 32, 185-188.

[4] Puxeddu, R., Berlucchi, M., Ledda, G.P., Parodo, G., Farina, D. and Nicolai, P. (2006) Lobular Capillary Hemangioma of the Nasal Cavity: A Retrospective Study on 40 Patients. American Journal of Rhinology, 20, 480-484. https://doi.org/10.2500/ajr.2006.20.2878

[5] Mills, S.E., Gaffey, M.J. and Frierson, H.F. (2000) Atlas of Tumor Pathology. Tumors of the Upper Aerodigestive Tract and Ear, 3rd Edition, Armed Forces Institute of Pathology, Washington DC, 243-271.

[6] Bhattacharyya, N., Wenokur, R.K. and Goodman, M.L. (1997) Endoscopic Excision of a Giant Pyogenic Granuloma of the Nasal Cavity Caused by Nasal Packing. Rhinology, 35, 44-45.

[7] Sheen, T.S., Ko, Y.J. and Hsu, Y.H. (1997) Pyogenic Granuloma-An Uncommon Complication of Nasal Packing. American Journal of Rhinology, 11, 225-227. https://doi.org/10.2500/105065897781751839

[8] Choudhary, S., Mackinnon, C.A., Morrissey, G.P. and Tan, S.T. (2005) A Case of Giant Nasal Pyogenic Granuloma Gravidarum. Journal of Craniofacial Surgery, 16, 319-321. https://doi.org/10.1097/00001665-200503000-00022

[9] Nedev, P. (2008) Lobular Capillary Haemangioma of the Nasal Cavity in Children. Trakia Journal of Sciences, 6, 63-67.

[10] Genc, S., Kurkcuoglu, S.S., Karabulut, H., Acar, B., Tuncel, U. and Degerli, S. (2009) Giant Lobular Capillary Hemangioma of the Nasal Septum. Turkish Journal of Medical Sciences, 39, 325-328.

[11] Archontaki, M., Stamou, A.K., Hajiioannou, J.K., Kalomenopoulou, M., Korkolis, D.P. and Kyrmizakis, D.E. (2008) Cavernous Haemangioma of the Left Nasal Cavity. Acta Otorhinolaryngologica Italica, 28, 309-311.

[12] Akiner, M.N., Akturk, M.T., Demirtas, M. and Atmis, E.O. (2011) Intraosseous Cavernous Hemangioma of Inferior Turbinate: A Rare Case Report. Case Reports in Otolaryngology, 431365.

[13] Budiman, B.J. and Octiza, R. Capillary Haemangioma of the Nasal Septum.

[14] Poncet, A. and Dor, L. (1897) Botyromycose Humaine. Rev Chir (Paris), 18, 996.

[15] Kapella, M., Panosetti, E., Rombaux, P., Delos, M. and Weynand, B. (2001) Lobular Capillary Haemangioma of the Nasal Cavity: Observation of Three Specific Cases. Acta Otorhinolaryngol Belg, 55, 241-246.

[16] Pagliai, K.A. and Cohen, B.A. (2004) Pyogenic Granuloma in Children. Pediatric Dermatology, 21, 10-13.

[17] Burlucchi, M., Peruzzi, B. and Farina, D. (2010) Lobular Capillary Hemangioma (LCH). Archives of Otolaryngology-Head \& Neck Surgery, 136, 1141-1143.

[18] Iwata, N., Hattori, H., Nakagawa, T. and Tsujimura, T. (2002) Hemangioma of the Nasal Cavity a Clinicopathologic Study. Auris Nasus Larynx, 29, 335-339.

[19] Chi, T.H., Yuan, C.H. and Chien, T.S. (2014) Lobular Capillary Hemangioma of the Nasal Cavity: A Retrospective Study of 15 Cases in Taiwan. Balkan Medical Journal, 31, 69-71. 
[20] Kataragama, Y.G., Howarth, K., Steel, P.R.M. and Spencer, M.G. (2002) Lobular Capillary Haemangioma of the Nasal Vestibule: A Rare Entity. International Journal of Pediatric Otorhinolaryngology, 66, 71-75. https://doi.org/10.1016/S0165-5876(02)00207-0

[21] Jones, J.E., Nguyen, A. and Tabaee, A. (2000) Pyogenic Granuloma (Pregnancy Tumor) of the Nasal Cavity. A Case Report. The Journal of Reproductive Medicine, $45,749-753$.

[22] Lee, D.G., Lee, S.K., Chang, H.W., Kim, J.Y., Lee, H.J., Lee, S.M., Kwon, J.H., et al. (2010) CT Features of Lobular Capillary Hemangioma of the Nasal Cavity. American Journal of Neuroradiology, 31, 749-754. https://doi.org/10.3174/ajnr.A1908

[23] Toida, M., Hasegawa, T., Watanabe, F., Kato, K., Makita, H., Fujitsuka, H., Kato, Y., et al. (2003) Lobular Capillary Hemangioma of the Oral Mucosa: A Clinicopathological Study of 43 Cases with a Special Reference to Immunohistochemical Characterization of the Vascular Elements. Pathology International, 53, 1-7.

https://doi.org/10.1046/j.1440-1827.2003.01434.x

[24] Kurtaran, H., Uraldi, C., Ark, N. and Aktas, D. (2006) Lobular Capillary Haemangioma of the Middle Turbinate. Acta Oto-Laryngologica, 126, 442-444. https://doi.org/10.1080/00016480500401001

[25] Lee, G., Suh, K., Lee, Y. and Kang, I. (2012) CT Findings in Two Cases of Lobular Capillary Haemangioma of the Nasal Cavity: Focusing on the Enhancement Pattern. Dentomaxillofacial Radiology, 41, 165-168. https://doi.org/10.1259/dmfr/85015314

[26] Lee, H.M., Lee, S.H. and Hwang, S.J. (2002) A Giant Pyogenic Granuloma in the Nasal Cavity Caused by Nasal Packing. European Archives of Oto-Rhino-Laryngology, 259, 231-233. https://doi.org/10.1007/s00405-001-0442-X

[27] Yang, B.T., Li, S.P., Wang, Y.Z., Dong, J.Y. and Wang, Z.C. (2013) Routine and Dynamic MR Imaging Study of Lobular Capillary Hemangioma of the Nasal Cavity with the Comparison to Inverting Papilloma. American Journal of Neuroradiology, 34, 2202-2207.

[28] El-Sayed, Y. and Al-Serhani, A. (1997) Lobular Capillary Hemangioma (Pyogenic Granuloma) of the Nose. The Journal of Laryngology \& Otology, 111, 941-945. https://doi.org/10.1017/S0022215100139027

[29] Smith, S.C., Patel, R.M., Lucas, D.R. and McHugh, J.B. (2013) Sinonasal Lobular Capillary Hemangioma: A Clinicopathologic Study of 34 Cases Characterizing the Potential for Local Recurrence. Head and Neck Pathology, 7, 129-134.

[30] Patil, P., Singla, S., Mane, R. and Jagdeesh, K.S. (2013) Nasal Lobular Capillary Haemangioma. Journal of Clinical Imaging Science, 3, 40.

https://doi.org/10.4103/2156-7514.119134 